\title{
Spondylodiscitis as a Complication of Transrectal Ultrasonography-Guided Prostate Biopsy: a Case Report
}

\author{
Di Fronzo V, Russo BF, Gargiulo F, Carlino V, Ferrara LA \\ Department of Clinical and Experimental Medicine, University of Naples Federico II, Naples, Italy
}

\begin{abstract}
:
A 61-year-old man presented with high fever, and severe back and abdominal pain following transrectal ultrasonography (TRUS)-guided prostate biopsy. Diagnosis of spondylodiscitis and psoas abscesses was made based on MRI images of the lumbar tract of the spine. Six-month broad-spectrum antibiotic treatment and immobilization with a girdle overcame the disease without any relapse at the 1-year follow-up.

Spondylodiscitis after TRUS-guided prostate biopsy is a rare event, which is not yet included as a major complication of the procedure. It is probably due to the presence of fluoroquinolone-resistant bacteria in faeces. It is, therefore, important to highlight this possibility and to stress the use of targeted antibiotic prophylaxis after rectal flora swabbing with selected antibiotics at sufficient concentrations to be effective.
\end{abstract}

Keywords: Spondylodiscitis, TRUS-guided prostate biopsy, antibiotic treatment

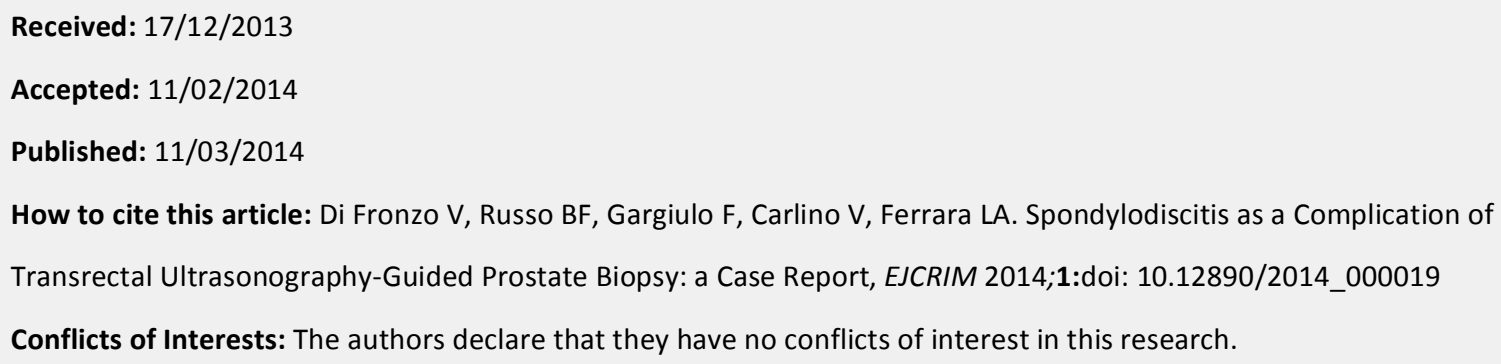




\section{Introduction}

Spondylodiscitis is a bacterial infection of the intervertebral disk and adjoining vertebrae. It is more frequently associated with conditions such as infections, malignant diseases or collagenosis.

Medical interventions may lead to iatrogenic spondylodiscitis either directly by inoculation or indirectly by haematogenous dissemination ${ }^{1}$ : these include spinal procedures, urogenital and vascular interventions and intravenous catheter use. Transrectal ultrasonography (TRUS)-guided prostate biopsy is the most frequently used tool for the diagnosis of prostate cancer. It is a relatively safe method, well tolerated by patients, although minor complications and, rarely, major complications such as sepsis do sometimes arise. Spondylodiscitis after TRUS-guided prostate biopsy is a rare event and few cases have been published ${ }^{2}$.

\section{Case report}

A 61-year-old male patient suffering back pain, abdominal pain, high fever and fatigue presented to the Department of Clinical Medicine at the University of Naples Federico II. He had undergone a TRUS-guided prostate biopsy a week before because of high serum prostate-specific antigen level. Antibiotic prophylaxis (ciprofloxacin $500 \mathrm{mg}$ ) had been performed a day before the procedure.

The histopathological features of 12 biopsy specimens showed benign prostate tissue.

High fever started with shivering one day after the prostate biopsy and back pain a few days later. He was treated with analgesic drugs and antibiotic (nebicine) for a week.

On physical examination, he complained of severe pain at the $L_{2}-L_{4}$ level of the spinal column and abdominal pain on palpation; neurological examination only showed bilateral lumbosacral nerve root irritation since pain was elicited when the legs were raised to an angle of almost $30^{\circ}$ (positive Lasègue's sign bilaterally, particularly on the right). The patient still had septic fever (peak temperature $40^{\circ} \mathrm{C}$ ).

Laboratory tests showed neutrophilic leucocytosis (white cells $12,400 / \mathrm{ml}$ and neutrophils $9,900 / \mathrm{ml}$ ) and other positive signs of general inflammation (high level of C-reactive protein (CRP), $17.8 \mathrm{mg} / \mathrm{dl}$; normal values, $0-5 \mathrm{mg} / \mathrm{dl}$ ). Serological tests for Brucella and Salmonella; purified protein derivative (PPD) test for tuberculosis; and blood and urine cultures were normal.

Chest X-ray and echocardiography were negative. Lumbosacral spine X-rays showed marked reduction in the height of the $L_{1}-L_{3}$ discs.

Abdominal ultrasound showed abscesses in the middle third of the right ileopsoas muscle $(36 \times 34 \times 51$ $\mathrm{mm})$ and in the proximal third of the left ileopsoas muscle $(35 \times 20 \mathrm{~mm})$ as well as hypoechogenicity of the middle lobe of prostate. 


\section{European Journal \\ of Case Reports in \\ Internal Medicine}

Thereafter, the patient underwent MRI, with contrast enhancement, of the lumbosacral spine.

Pre- and post-contrast multiplanar images obtained by T1-T2-weighted turbo spin echo and short $\mathrm{TI}$ inversion recovery (STIR) sequences showed widespread impregnation of the upper portion of $L_{3}$ and the lower portion of $L_{2}$ involving the epidural anterior tissue and lateral recesses bilaterally, compressing the spinal roots as well as the psoas muscle where abscesses were detected (Fig. 1). The patient refused to give consent for a biopsy.

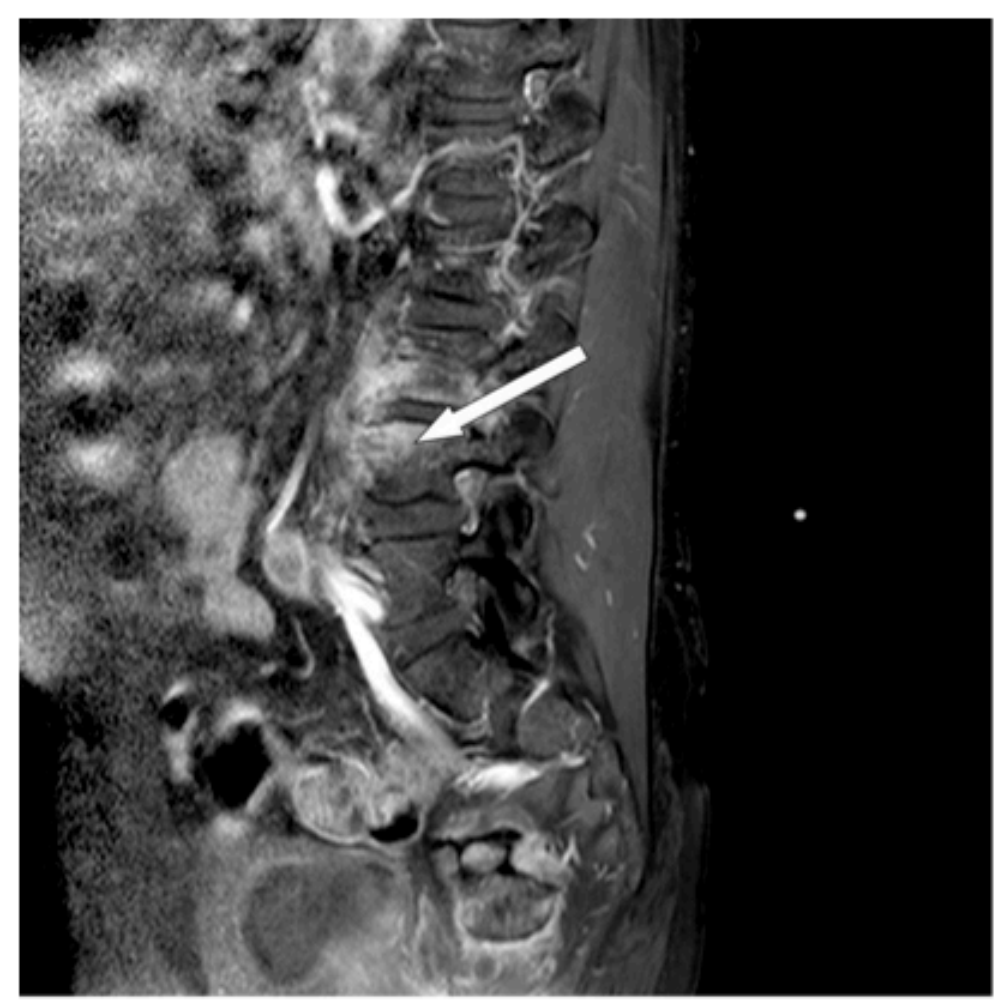

Figure 1: Pre- and post-contrast multiplanar images obtained by T1T2-weighted turbo spin echo and STIR sequences showed widespread impregnation of the upper portion of $L_{3}$ and the lower portion of $L_{2}$ involving the epidural anterior tissue and lateral recesses bilaterally, compressing the spinal roots as well as the psoas muscle where abscesses were detected.

Broad-spectrum antibiotic therapy, using rifampicin $600 \mathrm{mg}$ i.v., ceftazidime $2 \mathrm{~g}$ twice a day i.v. and teicoplanin $400 \mathrm{mg}$ i.v., was then started for six weeks. The patient also started to wear a metallic girdle.

Fever remitted 24 hours after starting antibiotic therapy. Fifteen days later, a new abdominal 


\section{European Journal \\ of Case Reports in \\ Internal Medicine}

ultrasound examination showed resolution of abscesses in the right and left ileopsoas.

Inflammatory markers (CRP, white blood cells and neutrophils), measured bi-weekly, declined over time. Also, abdominal pain, back pain and leg pain gradually reduced.

After 6 weeks of intravenous antibiotic therapy, with negative PPD and TIBI tests, the patient started treatment with ciprofloxacin $500 \mathrm{mg}$ orally twice daily and teicoplanin $400 \mathrm{mg} \mathrm{fl} \mathrm{i.m.} \mathrm{for} \mathrm{six} \mathrm{months.}$

Liver enzymes, renal function and inflammatory markers were monitored at weekly intervals.

At the end of the antibiotic treatment, the patient was asymptomatic and laboratory tests showed normal levels of white blood cells and CRP.

Another MRI of the lumbar spine revealed stabilized outcome of the previous infection, showing signs of spondylosis associated with structural irregularities of the cortical bone of $\mathrm{L}_{3}-\mathrm{L}_{4}$.

The discs included in the $L_{1}-L_{5}$ section had undergone a degenerative process (Fig. 2).

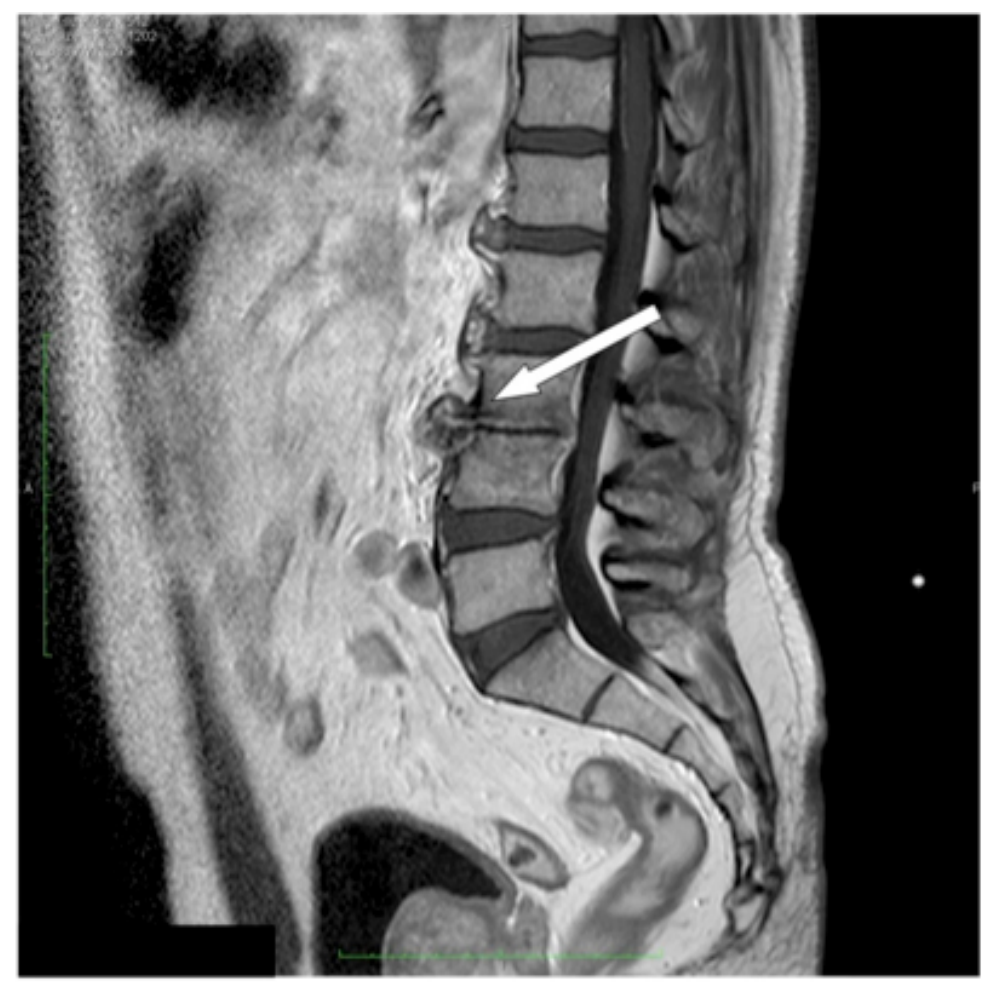

Figure 2: MRI of the lumbar spine performed 1 year later revealed stabilized outcome of the previous infection characterized by spondylosis associated with structural irregularities of the cortical bone of $L_{3}-L_{4}$. The discs included in the section $L_{1}-L_{s}$ had undergone a degenerative process.

\section{Discussion}




\section{European Journal}

of Case Reports in

Internal Medicine

TRUS-guided prostate needle biopsy is the ideal method to obtain prostate specimens for histological analysis and is, therefore, frequently used in clinical practice ${ }^{3}$. It is very rarely complicated by high fever/sepsis (0.6-6.6\%) ${ }^{1}$, usually caused by Escherichia coli, for inoculation of bacteria during the procedure when the needle passes through the contaminated rectum[3]. The use of antibiotic prophylaxis for TRUS-guided prostate biopsy significantly reduces the incidence of infective complications but does not completely eliminate the possibility. Although fluoroquinolones are still the first choice for prophylactic antibiotic therapy, their failure in this patient might be explained by the rather frequent finding of fluoroquinolone-resistant bacteria in faeces: $13 \%$ in the first report ${ }^{4}$ and $4 \%$ in a very recent paper ${ }^{5}$. Alternatively, we could suggest that treatment was not effective because it was given too early, even though it was performed according to the current guidelines.

In the present report, we describe a case of spondylodiscitis, a very severe and rare complication of TRUS-guided prostate biopsy not yet included in its list of major complications, which developed despite treating the patient with prophylactic antibiotic therapy at variance with previous cases.

On admission to the hospital, clinical presentation and laboratory analyses were typical of an acute infection. Diagnosis was suggested by the X-ray findings of the spinal column and subsequently validated by MRI. Other possible diagnoses (neoplastic infiltration of the bone, degenerative disk disease, intra-osseous disk herniation, primary spondylodiscitis in ankylosing spondylitis, rheumatoid arthritis) were excluded based on the clinical, laboratory and radiological findings. The clear evidence of the disease obtained with non-invasive techniques strongly supported starting long-term treatment with antibiotics even though a biopsy, which is considered the gold standard for diagnosing spondylodiscitis, had not been performed.

The patient recovered with antibiotic treatment ( 6 weeks of intravenous antibiotics followed by 5 months of oral medication) and immobilization (bed rest and girdle), according to current guidelines, and no recurrence of the disease was observed in the follow-up period, although it is reported as possible within six months. At 1-year follow-up patient does not suffer residual disabilities, despite it is reported in $1 / 3$ of the cases of spondylodiscitis.

The main message of this clinical case is that in patients at risk of fluoroquinolone resistance, prophylaxis of TRUS-guided prostate biopsy should be performed with carefully selected antibiotics at sufficient concentrations after rectal flora swabbing. 


\section{European Journal}

of Case Reports in

Internal Medicine

\section{Learning Points}

- Spondylodiscitis must be suspected in patients who complain of septic fever and back pain following TRUS-guided prostate biopsy.

- In these cases, early diagnosis and rapid initiation of treatment are critical to prevent or at least minimize this severe complication.

- Although prophylactic fluoroquinolone is the first choice in preventing acute bacterial prostatitis after TRUS-guided prostate biopsy, the presence of fluoroquinolone-resistant $E$. coli in the faeces must always be taken into account. Stool cultures for the detection of fluoroquinolone-resistant $E$. coli might be, therefore, suggested before TRUS-guided prostate biopsy.

\section{References}

1. Titlic M, Josipovic-Jelic Z. Spondylodiscitis, Bratis/ Lek Listy 2008;109:345-7.

2. Karapolat H, Akkoc $Y$, Arda B, Sesli E. Spondylodiscitis caused by sudden onset back pain following transrectal ultrasonography-guided prostate biopsy: a case report, Agri 2009;21:121-125.

3. Lucio E, Adesokan A, Hadjipavlou AG, Crow WN, Adegboyega PA. Pyogenic spondylo-diskitis: a radiologic/pathologic and culture correlation study, Arch Pathol Lab Med 2000;124:712-716.

4. Minamida S, Satoh T, Tabata K, Kimura M, Tsumura H, Kurosaka S, Matsumoto K, Fujita T, Iwamura M, Baba S. Prevalence of fluoroquinolone-resistant Escherichia coli before and incidence of acute bacterial prostatitis after prostate biopsy, Urology 2011;78:1235-1239.

5. Wagenlehner FM, Pilatz A, Waliszewski $P$, Weidner W, Johansen TE. Reducing infection rates after prostate biopsy, Nat Rev Urol 2014;11:80-86. 